\title{
Correction to: A network approach to investigating the key microbes and stability of gut microbial communities in a mouse neuropathic pain model
}

Guo-Jie Brandon-Mong ${ }^{1,2,3}$, Grace Tzun-Wen Shaw ${ }^{1}$, Wei-Hsin Chen ${ }^{4}$, Chien-Chang Chen ${ }^{4,5}$ and Daryi Wang ${ }^{1,3^{*}}$

\section{Correction to: BMC Microbiol 20, 295 (2020)}

https://doi.org/10.1186/s12866-020-01981-7

After publication of the original article [1], the authors have notified us that Fig. 5A was not the updated version; it was analyzed without simulations and permutation cutoff.

The original article can be found online at https://doi.org/10.1186/s12866020-01981-7.

* Correspondence: dywang@gate.sinica.edu.tw

${ }^{1}$ Biodiversity Research Center, Academia Sinica, 128 Academia Road, Sec. 2, Nankang, Taipei 11529, Taiwan

${ }^{3}$ Biodiversity Program, Taiwan International Graduate Program, Academia

Sinica and National Taiwan Normal University, Taipei, Taiwan

Full list of author information is available at the end of the article

(c) The Author(s). 2020 Open Access This article is licensed under a Creative Commons Attribution 4.0 International License, which permits use, sharing, adaptation, distribution and reproduction in any medium or format, as long as you give appropriate credit to the original author(s) and the source, provide a link to the Creative Commons licence, and indicate if changes were made. The images or other third party material in this article are included in the article's Creative Commons licence, unless indicated otherwise in a credit line to the material. If material is not included in the article's Creative Commons licence and your intended use is not permitted by statutory regulation or exceeds the permitted use, you will need to obtain permission directly from the copyright holder. To view a copy of this licence, visit http://creativecommons.org/licenses/by/4.0/. The Creative Commons Public Domain Dedication waiver (http://creativecommons.org/publicdomain/zero/1.0/) applies to the data made available in this article, unless otherwise stated in a credit line to the data. 
The correct Fig. 5A is presented below.

A

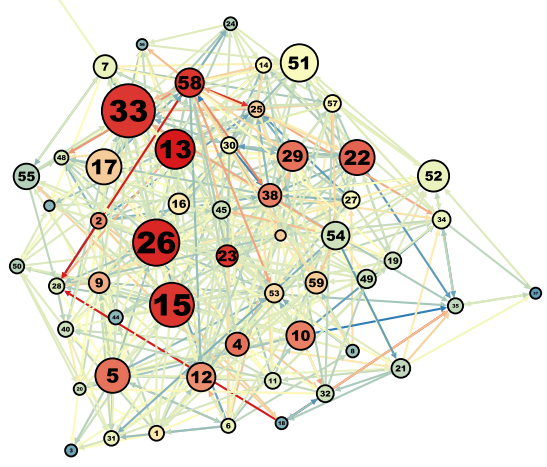

B

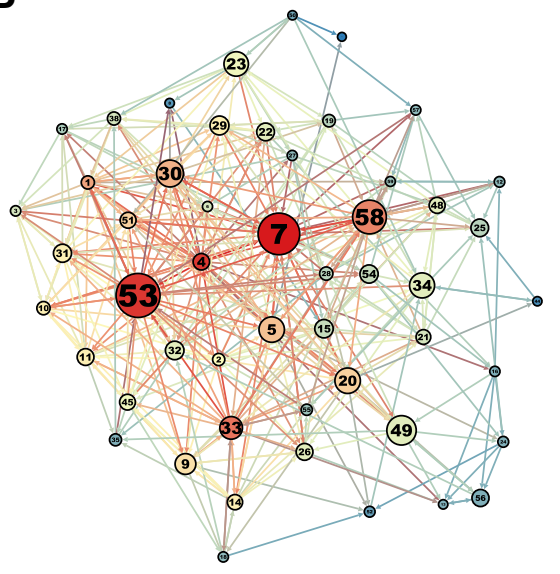

Interaction strength

\begin{tabular}{|l|l|}
\hline OTU & Taxonomy \\
\hline Otu01 & Lachnospiraceae_unclassified \\
\hline Otu02 & Bacteroidales_S24-7 \\
\hline Otu03 & Bacteria_unclassified \\
\hline Otu04 & Bacteroidales_unclassified \\
\hline Otu05 & Ruminococcaceae_unclassified \\
\hline Otu06 & Clostridiales_unclassified \\
\hline Otu07 & Ruminococcaceae_uncultured \\
\hline Otu08 & Lachnospiraceae_Incertae_Sedis \\
\hline Otu09 & Firmicutes_unclassified \\
\hline Otu10 & Ruminococcaceae_Incertae_Sedis \\
\hline Otu11 & Lachnospiraceae_uncultured \\
\hline Otu12 & Mollicutes_RF9 \\
\hline Otu13 & Adlercreutzia \\
\hline Otu14 & Bacteroidetes_unclassified \\
\hline Otu15 & Erysipelotrichaceae_unclassified \\
\hline Otu16 & Coriobacteriaceae_unclassified \\
\hline Otu17 & Lactobacillus \\
\hline Otu18 & Moryella \\
\hline Otu19 & Clostridiales_Family_XIII_Incertae_Sedis \\
\hline Otu20 & Oscillibacter \\
\hline
\end{tabular}

\begin{tabular}{|l|l|}
\hline OTU & Taxonomy \\
\hline Otu21 & Acetanaerobacterium \\
\hline Otu22 & Bacteroides \\
\hline Otu23 & Barnesiella \\
\hline Otu24 & Coprobacillus \\
\hline Otu25 & Allobaculum \\
\hline Otu26 & Oscillospira \\
\hline Otu27 & Clostridium_Candidatus_Arthromitus \\
\hline Otu28 & Akkermansia \\
\hline Otu29 & Parabacteroides \\
\hline Otu30 & Mucispirillum \\
\hline Otu31 & Hydrogenoanaerobacterium \\
\hline Otu32 & Roseburia \\
\hline Otu33 & Ruminococcus \\
\hline Otu34 & Candidate_division_TM7 \\
\hline Otu35 & Lachnospira \\
\hline Otu36 & Lactobacillaceae_unclassified \\
\hline Otu37 & Enterococcus \\
\hline Otu38 & Parasutterella \\
\hline Otu39 & Pseudomonas \\
\hline Otu40 & Porphyromonadaceae_unclassified \\
\hline
\end{tabular}

\begin{tabular}{ccc}
\hline$\vec{\Delta}$ & $\Delta$ \\
$-9.5 \mathrm{E}-04$ & $\mathbf{0 . 0}$ & $7.5 \mathrm{E}-04$
\end{tabular}

\begin{tabular}{|l|l|}
\hline OTU & Taxonomy \\
\hline Otu41 & Alcaligenes \\
\hline Otu42 & Weissella \\
\hline Otu43 & Robinsoniella \\
\hline Otu44 & Acinetobacter \\
\hline Otu45 & Erysipelotrichaceae_Incertae_Sedis \\
\hline Otu46 & Lactobacillales_unclassified \\
\hline Otu47 & Olsenella \\
\hline Otu48 & Bifidobacterium \\
\hline Otu49 & Streptococcus \\
\hline Otu50 & Clostridia_unclassified \\
\hline Otu51 & Clostridium_unclassified \\
\hline Otu52 & Escherichia \\
\hline Otu53 & Staphylococcus \\
\hline Otu54 & Desulfovibrio \\
\hline Otu55 & Mollicutes_unclassified \\
\hline Otu56 & Actinobacteria_unclassified \\
\hline Otu57 & Eubacterium \\
\hline Otu58 & Turicibacter \\
\hline Otu59 & Peptococcaceae \\
\hline & \\
\hline
\end{tabular}

-The update of this figure does not change the results, discussion, and conclusion of the original article. We apologize for the inconvenience caused.

\section{Author details}

${ }^{1}$ Biodiversity Research Center, Academia Sinica, 128 Academia Road, Sec. 2, Nankang, Taipei 11529, Taiwan. ${ }^{2}$ Department of Life Science, National Taiwan Normal University, Taipei, Taiwan. ${ }^{3}$ Biodiversity Program, Taiwan International Graduate Program, Academia Sinica and National Taiwan Normal University,

Taipei, Taiwan. ${ }^{4}$ Institute of Biomedical Sciences, Academia Sinica, Taipei, Taiwan. ${ }^{5}$ Taiwan International Graduate Program in Molecular Medicine, National Yang-Ming University, Academia Sinica, 128 Academia Road, Sec. 2, Nankang, Taipei 11529, Taiwan.

Published online: 28 December 2020

\section{Reference}

1. Brandon-Mong G-J, et al. A network approach to investigating the key microbes and stability of gut microbial communities in a mouse neuropathic pain model. BMC Microbiol. 2020;20:295. https://doi.org/10. 1186/s12866-020-01981-7. 